\title{
Determination of ppb Levels of Tryptophan Derivatives by Capillary Electrophoresis with Homogeneous Liquid-Liquid Extraction and Sweeping Method
}

\author{
Yoshitaka TAKAgAI and Shukuro IgARASHI* \\ Department of Materials Science, Faculty of Engineering, Ibaraki University; 4-12-1 Nakanarusawa, Hitachi, Ibaraki \\ 316-8511, Japan. Received September 27, 2002; accepted December 12, 2002
}

\begin{abstract}
Tryptophan (Trp) derivatives were selectively separated from 24 kinds of amino acid mixture solutions. Capillary electrophoresis (CE) with UV detection using the described pretreatment system was able to separate and determine 4 kinds of Trp derivatives: 5-hydroxy L-tryptophan, 5-methyl L-tryptophan, 1-methyl L-tryptophan and L-tryptophan. The pretreatment system used a combination of a homogeneous liquid-liquid extraction and a sweeping method, the two are different kinds of powerful preconcentration/separation methods. Trp derivatives were thus separated by two different separation systems. First, these derivatives were selectively extracted at $57-100 \%$ by a homogeneous liquid-liquid extraction; secondly, they were respectively separated by micellar electrokinetic chromatography (MEKC) with a sweeping effect. The detection limits following complete separation of the Trp derivatives were $10^{-8} \mathrm{moll}^{-1}$ levels, respectively. The proposed method provided a more than 10fold the improvement in sensitivity compared with fluorescence labeling/fluorophotometric analysis; the proposed method was also applied to the component analysis of nutritious medicine.
\end{abstract}

Key words tryptophan derivative; homogeneous liquid-liquid extraction; sweeping method

Trp compounds are very important, not only in being a kind of essential amino acid but also in producing many metabolites such as serotonin. However, a breathing difficulties and skin diseases were reported by ingesting synthesized Trp. ${ }^{1)}$ Liquid chromatography ${ }^{2,3)}$ and capillary electrophoresis $(\mathrm{CE})^{4-6)}$ are known as general analytical methods, but coexisting amino acids and amines interfere with the Trp analysis. In an attempt to solve this problem, the authors previously reported ${ }^{7)}$ a homogeneous liquid-liquid extraction/fluorophotometric analysis using fluorescamine (FLA) which is a labeling reagent. Several serious problems, however, remain to be solved. One of them is non-selectivity toward several derivatives such as methyl derivatives and/or hydroxy derivatives.

In this study, a high performance separation system is presented for the analysis of several derivatives. The system was developed by combining an off-line preconcentration method and an on-line one. Both the preconcentration and separation methods use a perfluoro surfactant as a separation medium. Combining two different kinds of pretreatment methods permits an increase in the selectivity for specific compounds.

The homogeneous liquid-liquid extraction with perfluorooctanoate ion $^{8-10)}$ as the first-step separation system is the off-line preconcentration and separation method. The method utilizes the $\mathrm{pH}$-dependent phase separation phenomenon from a homogenous solution, and can powerfully and rapidly concentrates (100-100000 fold) down to micro-volume levels ( $\mu 1$ levels) within a few minutes. A sweeping method ${ }^{11-13)}$ as the second-step is an on-line concentration and separation method for CE. It is based on micellar electrokinetic chromatography (MEKC), ${ }^{14-16)}$ which is a mode of separation in $\mathrm{CE}$, under acidic conditions. In a negligible electroosmotic flow, neutral substances were efficiently concentrated and separated by micelles as a pseudostationary phase.

In this paper, the selective separation of Trp derivatives from many amino acid mixtures is described using the twostep separation system.

\section{Experiment}

Reagents Amino acids (alanine, arginine, asparagine, aspartic acid, cysteine, cystine, glutamic acid, glutamine, glycine, histidine, isoleucine, leucine, lysine, methionine, phenylalanine, serine, threonine, tryptophan, tyrosine, valine) were of $\mathrm{L}$-form and were analytical reagent grade. They were purchased from Kanto Chemical Co. (Tokyo, Japan). The 5-methyl tryptophan and 5-hydroxy tryptophan were purchased from Wako (Osaka, Japan) and 1-methyl tryptophan was purchased from Aldrich Japan (Tokyo). Perfluorooctanoic acid (HPFOA) and perfluorooctanesulfonic acid (HPFOS) were from DAIKIN (Osaka), and these aqueous solutions were used and neutralized with lithium hydroxide. FLA was prepared by dissolving in acetone. All other chemicals were of analytical reagent grade from Kanto Chemical Co., unless otherwise noted.

Apparatus CAPI-3200 (Otsuka Electronics, Osaka) was used as the capillary electrophoresis system. A $75-\mu \mathrm{m}$ I.D. fused-silica capillary (Otsuka Electronics) of 50-cm total length was used. Trp derivatives were detected and determined at $254 \mathrm{~nm}$ by a multi-photodiode array detector.

Experimental Procedure Twenty milliliters of sample solution containing amino acids, $1 \mathrm{ml}$ of borax buffer $(\mathrm{pH} \mathrm{9.4)} \mathrm{and} 1 \mathrm{ml}$ of an acetone solution of excessive FLA was placed in a 50-ml cylindrical glass vial. After gentle shaking, the mixture was allowed to stand at room temperature $\left(25^{\circ} \mathrm{C}\right)$ for $5 \mathrm{~min}$. One $\mathrm{ml}$ of $0.1 \mathrm{~mol}^{-1}$ perfluorooctanate ion (PFOA) was added to the solution. When $10 \mathrm{ml}$ of $6 \mathrm{~mol}^{-1} \mathrm{HCl}$ was added, the microliter levels of a water-immiscible liquid phase appeared from the homogeneous solution which was composed of water/acetone/PFOA ${ }^{-}$. The mixture was then centrifuged at $2000 \mathrm{rpm}$ for $2 \mathrm{~min}$. In the separating phase, analytical targets in the sample solution were extracted into the sedimented phase. Thirty microliters of the sedimented liquid phase was removed by micro-syringe and placed in a CE vial, then $30 \mu \mathrm{l}$ of dimethyl sulfoxide (DMSO) was added to the vial. This sample was hydrostatically injected into a capillary for $200 \mathrm{~s}$ from a height of $25 \mathrm{~mm}$. Under the conditions of running buffer (0.2 $\mathrm{mol}^{-1}$ perfluorooctanesulfonic ion, $40 \%$ DMSO and $0.1 \mathrm{~mol}^{-1} \mathrm{pH} 2.3$ (phosphoric acid)), a voltage of $-20 \mathrm{kV}$ was applied.

\section{Results and Discussion}

FLA Derivative Reaction Five Trp derivatives were used: 5-hydroxy L-tryptophan, $N$-methyl L-tryptophan, 5methyl L-tryptophan, 1-methyl L-tryptophan and L-tryptophan, as a model compound (shown in Fig. 1). FLA was used as a labeling reagent for these reasons: (i) the derivative reaction can be performed in water, (ii) the reactants were hydrophobic compounds and (iii) the derivative reaction is 


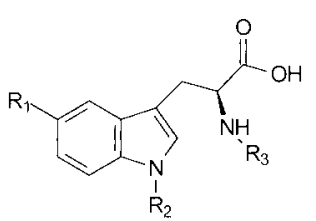

\begin{tabular}{lccc}
\hline \multicolumn{1}{c}{ Trp derivatives } & $\mathrm{R}_{1}$ & $\mathrm{R}_{2}$ & $\mathrm{R}_{3}$ \\
\hline 5-methyl L-tryptophan & $\mathrm{CH}_{3}$ & $\mathrm{H}$ & $\mathrm{H}$ \\
1-methyl L-tryptophan & $\mathrm{H}$ & $\mathrm{CH}_{3}$ & $\mathrm{H}$ \\
L-tryptophan & $\mathrm{H}$ & $\mathrm{H}$ & $\mathrm{H}$ \\
5-hydoroxy L-tryptophan & $\mathrm{OH}$ & $\mathrm{H}$ & $\mathrm{H}$ \\
N-methyl L-tryptophan & $\mathrm{H}$ & $\mathrm{H}$ & $\mathrm{CH}_{3}$ \\
\hline
\end{tabular}

Fig. 1. Structure Formula of Trp Derivatives

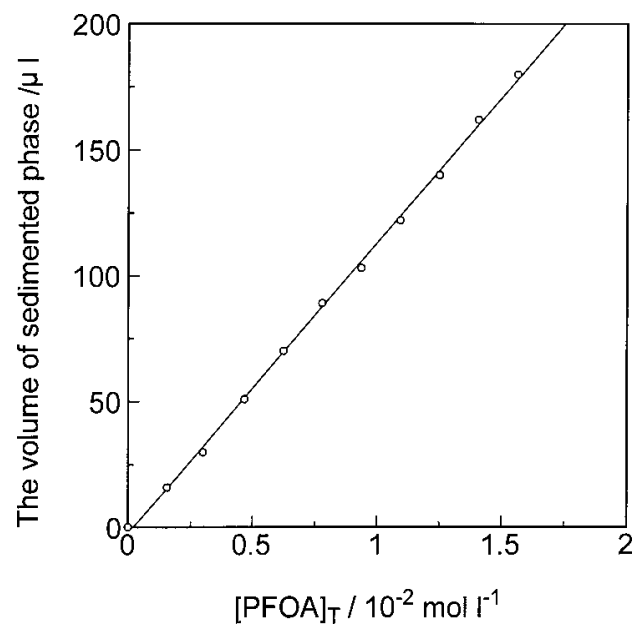

Fig. 2. Relationship between the Volume of Sedimented Phase and the Added PFOA

Total volume; $33 \mathrm{ml}$ at $25^{\circ} \mathrm{C}$, [acetone $]_{\mathrm{T}}=3 \mathrm{vol} \%,[\mathrm{HCl}]_{\mathrm{T}}=1.82 \mathrm{~mol}^{-1}$.

rapid and quantitative. Optimized conditions are shown in the section on experimental procedure.

Homogeneous Liquid-Liquid Extraction Homogeneous liquid-liquid extraction with $\mathrm{PFOA}^{-}$is based on a $\mathrm{pH}-\mathrm{de}$ pendent phase separation phenomenon. By homogeneous liquid-liquid extraction, the microliter levels of a water-immiscible liquid phase appear from the homogeneous solution. Analytical targets in the sample solution were extracted into the sedimented phase. The phase separation phenomenon was based on the acid dissociation of $\operatorname{PFOA}^{-}\left(\mathrm{p} K_{\mathrm{a}}=1.01^{8)}\right)$, and the $\mathrm{pH}$ was adjusted to below 1 using $6 \mathrm{~mol}^{-1}$ of hydrochloric acid. The relationship between PFOA concentration and volume of the sedimented phase is shown in Fig. 2. The sedimented phase volume was in proportion to the total concentration of $\mathrm{PFOA}^{-}$, making it possible to optionally establish the volume of the sedimented phase. Because of ease of handling of the sedimented phase, the total $\mathrm{PFOA}^{-}$concentration was determined to be $3 \times 10^{-3} \mathrm{~mol}^{-1}$ which was obtained in $30 \mu \mathrm{l}$ of this phase.

In the homogeneous liquid-liquid extraction with $\mathrm{PFOA}^{-}$, it is necessary for sedimented phase production to add a micro volume of a water-miscible organic solvent such as acetone, tetrahydorofuran or ethanol, etc. In this method, acetone was also used as a FLA solubilizer.
Table 1. Extraction Percentage of FLA-Tryptophan Derivatives in Homogeneous Liquid-Liquid Extraction Method

\begin{tabular}{lcc}
\hline \hline \multicolumn{1}{c}{ FLA-amino acid } & Extraction percentage ${ }^{a)}, \%$ & $\mathrm{RSD}^{b)}, \%$ \\
\hline L-Tryptophan & 81.1 & 2.2 \\
1-Methyl L-tryptophan & 83.3 & 5.8 \\
5-Methyl L-tryptophan & 100 & 4.3 \\
5-Hydroxy L-tryptophan & 57.5 & 4.9 \\
N-Methyl L-tryptophan & 2.3 & 3.1 \\
\hline
\end{tabular}

a) $[\text { tryptophan derivatives }]_{\mathrm{T}}=7.5 \times 10^{-7} \mathrm{~mol} \mathrm{l}^{-1}$. b) Five determinations.

Extraction and Separation of Trp Derivatives in Homogeneous Liquid-Liquid Extraction The extraction percentages of FLA-Trp derivatives are shown in Table 1. There are great differences in distribution due to differences in the hydrophilicity of the substituents. Moreover, FLA is a specific derivative reagent for primary amines, and a reactive intermediate is decomposed in an acidic condition. Therefore, the extraction percentage of $N$-methyl-L-tryptophan which is not a primary amine was very low compared to the other Trp derivatives. Moreover, the extraction percentages of 19 kinds of FLA-amino acids (L-form) except for Trp derivatives (alanine, arginine, asparagine, aspartic acid, cysteine, cystine, glutamic acid, glutamine, glycine, histidine, isoleucine, leucine, lysine, methionine, phenylalanine, serine, threonine, tyrosine, valinealanine) were below $0.4 \%$. Our previous report ${ }^{7)}$ assumed the following to be the reason for this: The FLA-amino acids (except for Trp derivatives) were decomposed under the acidic condition which is present in the homogeneous liquid-liquid extraction procedure, however, the Trp-FLA derivatives were stable under this strongly acidic condition. This stability is acquired by the conformation based on the interaction of Trp and FLA and the intermolecular hydrogen bond of the carboxyl groups between Trp and FLA.

The extracted tryptophan was stable for at least $40 \mathrm{~min}$ following an addition of $1.8 \mathrm{~mol}^{-1}$ hydrochloric acid.

Furthermore, in the case of non-derivatization without using FLA, the extraction percentages of all free amino acids were below $2 \%$.

CE Sweeping Separation of Trp-FLA Derivatives The sweeping concentration method ${ }^{11-13)}$ is based on MEKC under a negligible electroosmotic flow. Analytes were concentrated by sweeping the micelle migration based on electrophoresis. Three kinds of surfactant, $\mathrm{PFOA}^{-}, \mathrm{PFOS}^{-}$and sodium dodecyl sulfate (SDS), were examined. In SDS, the sedimented phase was difficult to solubilize in the running buffer, since the phase was very hydrophobic being composed of the perfluoro compound (HPFOA). With $\mathrm{PFOA}^{-}$, a needle crystal precipitated under the acidic condition $\left(\mathrm{p} K_{\mathrm{a}}=\right.$ 1.01). Since the $\mathrm{p} K_{\mathrm{a}}$ of the PFOS $^{-}$is nearly zero, the sweeping concentration was possible over the entire $\mathrm{pH}$ range. PFOS $^{-}$showed an on-line concentration effect even under acidic conditions, also, $\mathrm{PFOS}^{-}$has the same length of fluorocarbon as HPFOA. As a result, the sample solubilization was improved by using $\mathrm{PFOS}^{-}$.

The optimized $\mathrm{pH}$ was in the range of 2 to 3 , which suppressed the electroosmotic flow. Under this $\mathrm{pH}$ condition, the on-line concentration was effectively performed. The experiments were performed at $\mathrm{pH}$ 2.3. Above the optimized $\mathrm{pH}$, peaks did not appear. It is believed that HPFOA, which is in 


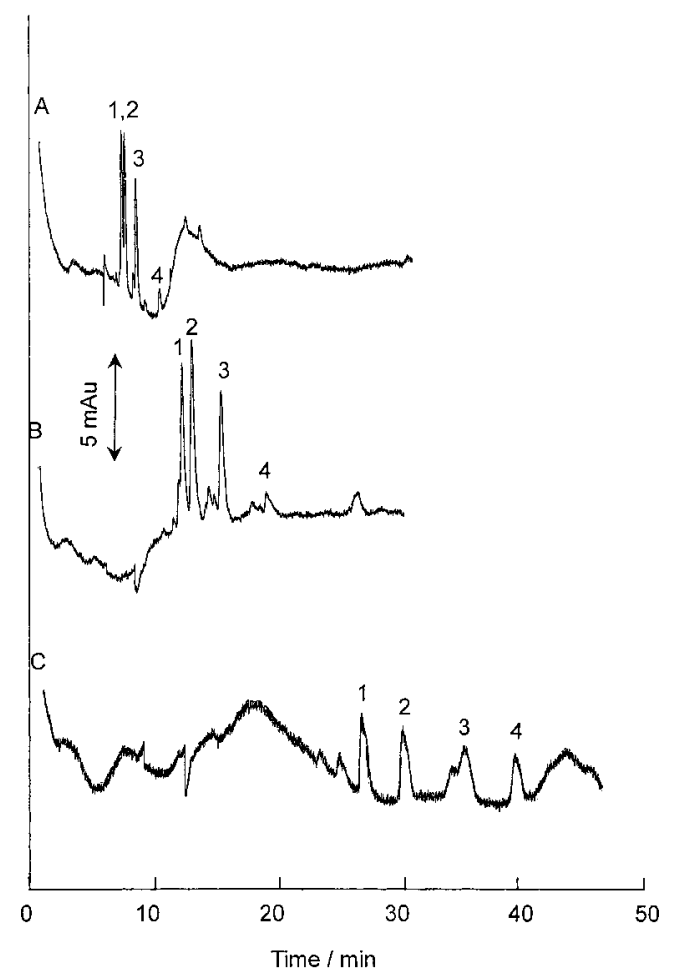

Fig. 3. Influence of DMSO on the Separation of Trp Derivative

A: DMSO $30 \%$, B: DMSO $40 \%$, C: DMSO $50 \%$. Running buffer condition 0.2 moll $^{-1}$ PFOS, pH 2.3 (phosphoric acid). [amino acid] ${ }_{\mathrm{T}}=3 \times 10^{-6} \mathrm{moll}^{-1}$, detection wavelength; $254 \mathrm{~nm}$. 1: 5-methyl L-tryptophan, 2: 1-methyl L-tryptophan, 3: L-tryptophan, 4: 5-hydroxy L-tryptophan.

the sample solution, dissociates $\mathrm{H}^{+}$and forms the micelle. Therefore, the sample zone moved on the capillary as a whole and could not be concentrated. On the other hand, the electric current was violently disordered below the optimized $\mathrm{pH}$. It is believed that this disorder was caused by an increase in salt concentration in the running buffer. In an optimized $\mathrm{pH}$, the stability of the Trp-FLA derivatives was examined in acidic running buffer, and there was no acid decomposition in the acidic buffer.

DMSO was added to the running buffer as an organic modifier $^{17)}$ to help solubilize the sedimented phase. The volume added was examined in the range of 30 to $60 \%$ (shown in Fig. 3). The Trp derivatives were separated by this DMSO addition to the running buffer. The DMSO limited a distribution of FLA-Trp to the PFOS micelles. It was difficult for 30\% DMSO (Fig. 3A) to separate 5-methyl Trp. (Peak 1) and 1-methyl Trp. (Peak 2); however, this separation was improved using 40\% DMSO (Fig. 3B). When 50\% DMSO was added (Fig. 3C), the peaks were broad and their intensities had decreased. Moreover, the peaks did not appear within one hour following addition of $60 \% \mathrm{DMSO}$, and it is considered that DMSO prevented distribution of FLA-Trp to the micelles. Thus, an effective concentration and a complete separation were achieved under a $40 \%$ DMSO condition. Influence of DMSO on the PFOS micelle formation is shown in Fig. 4. The value of CMC was determined by conductometry. A CMC of $\mathrm{PFOS}^{-} \mathrm{Li}^{+}$salt was reported ${ }^{18)}$ as a $6.3 \times$ $10^{-3} \mathrm{~mol} \mathrm{l}^{-1}\left(25^{\circ} \mathrm{C}\right.$, surface tension measurement) by Shinoda et al. In this study, CMC was obtained $8.1 \times 10^{-3} \mathrm{~mol} \mathrm{l}^{-1}$ $\left(17^{\circ} \mathrm{C}\right.$, conductivity measurement). This difference in results was considered due to the difference in experimental condi-

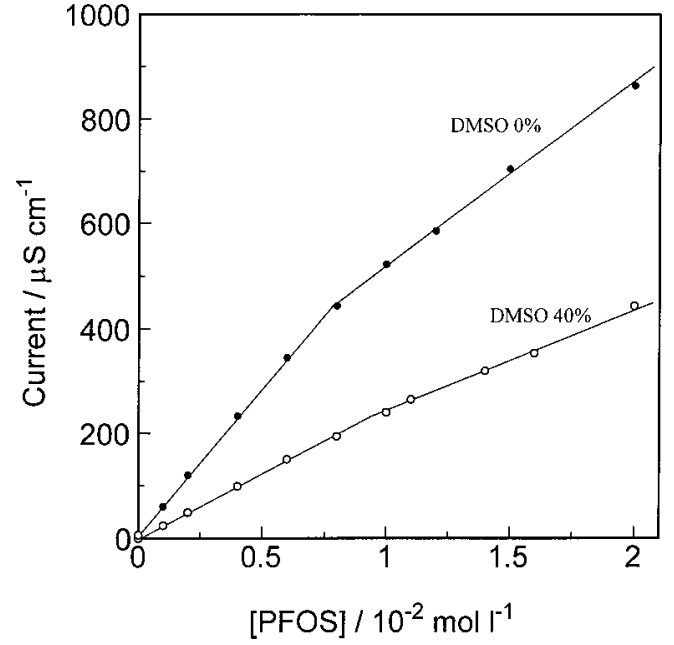

Fig. 4. Influence of DMSO on the PFOS Micelle Formation

- Non addition of DMSO, $\bigcirc$; addition of $40 \%$ DMSO

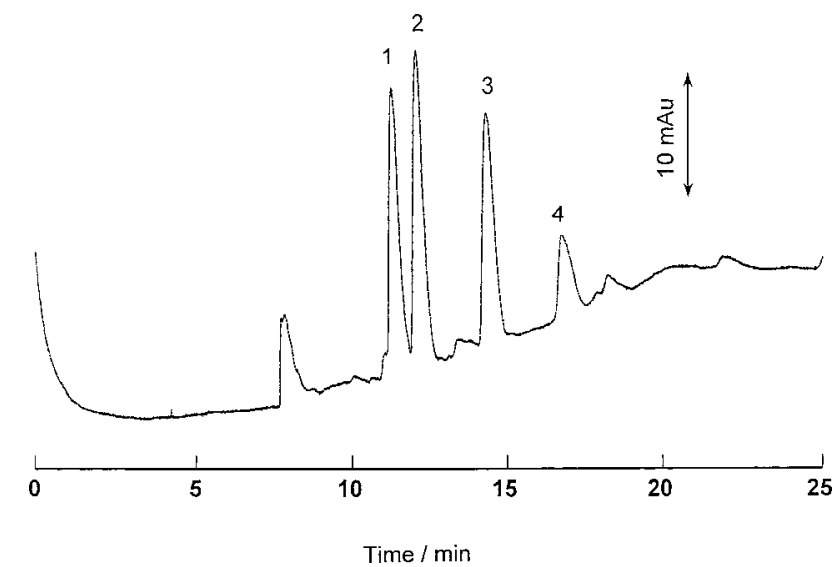

Fig. 5. Electropherogam after the Two-Step Separation from 24 Kinds of Amino Acid Mixed Solutions

[amino acid $]_{\mathrm{T}}=3 \times 10^{-6} \mathrm{moll}^{-1}$, total concentration factor: 5500 -fold, detection wavelength; $254 \mathrm{~nm}$. Running buffer condition; $0.2 \mathrm{moll}^{-1}$ PFOS, $40 \%$ DMSO, pH 2.3 (phosphoric acid). 1: 5-Methyl L-tryptophan, 2: 1-methyl L-tryptophan, 3: L-tryptophan, 4: 5-hydroxy L-tryptophan.

tions, especially temperature. Addition of DMSO was also found to restrain the conductivity. Therefore, DMSO is thought to be expected a functional material which limits the increase of electronic current on a capillary. When the condition was $40 \%$ DMSO, the CMC of PFOS was $9.1 \times 10^{-3}$ mol $1^{-1}\left(17^{\circ} \mathrm{C}\right.$, conductivity measurement). PFOS was found able to form the micelles in a higher concentration ratio of polar organic solvent. In a water/DMSO mixed solution, the apparent $\mathrm{p} K_{\mathrm{a}}$ is higher than the true value $\left(\mathrm{p} K_{\mathrm{a}}=1.01\right)$ with respect to HPFOA. When the condition was $40 \% \mathrm{DMSO}$, the apparent $\mathrm{p} K_{\mathrm{a}}$ was 2.8 , thus, HPFOA in sample solution did not dissociate $\mathrm{H}^{+}$in the capillary. An electropherogram under the optimized condition is shown in Fig. 5. This sample solution included 24 kinds of amino acids. When the twostep separation was used, four Trp derivatives were completely separated.

Simultaneous Determination of Trp Derivatives Firstly, the injection time was examined from 20 to $250 \mathrm{~s}$ by a hydrostatic method. The peak heights and areas increased in proportion with the injection time $(20-200 \mathrm{~s})$. Moreover, when 
Table 2. Determination Ranges and Detection Limits

\begin{tabular}{|c|c|c|c|}
\hline Trp derivatives & Determination range $/ \mathrm{mol} \mathrm{l}^{-1}$ & $\mathrm{RSD}^{b)}, \%$ & Detection limit ${ }^{c)} / \mathrm{mol} \mathrm{l}^{-1}$ \\
\hline 5-Methyl L-tryptophan & $3.7 \times 10^{-7}-1.8 \times 10^{-5}$ & 2.7 & $1.74 \times 10^{-8}$ \\
\hline 1-Methyl L-tryptophan & $3.7 \times 10^{-7}-1.8 \times 10^{-5}$ & 2.5 & $5.09 \times 10^{-8}$ \\
\hline L-Tryptophan & $3.7 \times 10^{-7}-1.2 \times 10^{-5}$ & 2.3 & $4.68 \times 10^{-8}$ \\
\hline 5-Hydroxy L-tryptophan & $1.8 \times 10^{-7}-6.0 \times 10^{-6}$ & 2.6 & $9.10 \times 10^{-8}$ \\
\hline
\end{tabular}

a) Total concentration factor; 5500-fold. b) Five determinations (central value of calibration curve). c) $3 \sigma$.

Table 3. Comparison with Highly Sensitive Analytical Methods for Trp Only

\begin{tabular}{lccc}
\hline \hline Analytical method & Linear range/mol 1 & RSD $^{a)}, \%$ & Detection of limit $^{b /} / \mathrm{mol} \mathrm{l}^{-1}$ \\
\hline CE/UV using the proposed method & $3.7 \times 10^{-7}-1.2 \times 10^{-5}$ & 2.3 & $4.7 \times 10^{-8}$ \\
Fluorescence analysis using the homogeneous liquid-liquid extraction ${ }^{c)}$ & $2.0 \times 10^{-6}-5.0 \times 10^{-5}$ & 1.2 & $7.0 \times 10^{-7}$ \\
CE/laser-induced fluorescence detectection ${ }^{d)}$ & - & - & $6.0 \times 10^{-8}$ \\
\hline
\end{tabular}

a) Five determinations. b) $3 \sigma . \quad$ c) S. Oshite et al., Analyst, 126, 703 (2001). d) T. Kaneta et al., J. Chromatogra. A, 805, 295 (1998).

samples were injected for more than $200 \mathrm{~s}$, the peaks were the broad and/or showed a tailing. The determination procedure was thus performed using a $200 \mathrm{~s}$ injection. The determination ranges and detection limits are shown in Table 2. Under optimized condition, the concentration-factor (volume ratio) was 5500 -fold and is explained ${ }^{11,19)}$ as follows. First, a sample solution was concentrated 1100 -fold by the homogeneous liquid-liquid extraction method (sample solution; $33 \mathrm{ml} \rightarrow$ sedimented phase; $30 \mu \mathrm{l}$ ), and the volume of the sedimented phase was diluted twice in the CE vial; this solution was then concentrated 10 -fold by the sweeping method. Other reports using sweeping method ${ }^{11-13)}$ achieved more than 10-fold preconcentration. However, our concentration was limited to 10 -fold. This reason is that there was a great difference in conductivity between each sample solution and the running buffer.

With respect to the determination ranges and detection limits, the proposed method was compared with the techniques of fluorophotometric analysis ${ }^{7)}$ and CE/laser-induced fluorescence detection. ${ }^{6}$ The results are shown in Table 3. The proposed method was able to determine wider than those of fluorescence analysis, because it has UV detection and does not affect the concentration quenching as occurs in fluorescence analysis. Regarding the detection limits, this method showed greater sensitivity compared with the fluorophotometric analysis using the preconcenration method and CE/laser-induced fluorescence detection. Since a UV detector is generally and widely used, we also used it. In the method described here, Trp derivatives were treated with fluorescence labeling. Therefore, if a more sensitive detector such as a fluorescence detector or a laser-induced fluorescence detector is used, the sensitivity could be even further increased.

Application to Real Sample The proposed method was applied to the analysis of Trp in a nutritious medical syrup. The syrup was composed of 10 essential amino acids (Lisoleucine, $30 \mathrm{mg}$; L-leucine, $34 \mathrm{mg}$; L-lysine, $37.5 \mathrm{mg}$; L-methionine, $30 \mathrm{mg}$; L-phenylalanine, $20 \mathrm{mg}$; L-threonine, $20 \mathrm{mg}$; L-tryptophan, $10 \mathrm{mg}$; L-valine, $30 \mathrm{mg}$ ) and taurine, vitamin $\mathrm{B}_{1}$, vitamin $\mathrm{B}_{2}$ and nicotineamide. The syrup was diluted 1000-fold, $0.1 \mu \mathrm{g}$ each of 5-hydroxy L-trp, 5-methyl L-Trp and 1-methyl L-Trp was added and the solution was analyzed

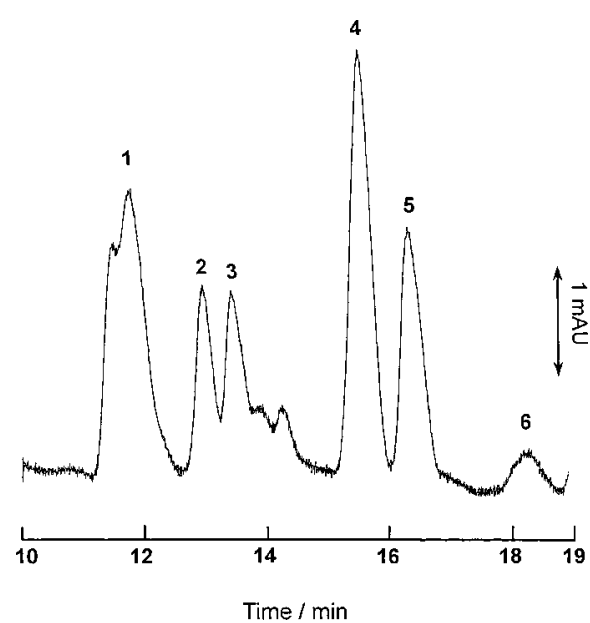

Fig. 6. Electropherogam of Diluted Nutritious Medical Syrup

Total concentration factor: 5500-fold, Detection wavelength; $254 \mathrm{~nm}$. Running buffer condition; $0.2 \mathrm{moll}^{-1}$ PFOS, 40\% DMSO, pH 2.3 (phosphoric acid). 1: FLA, 2: 5methyl L-tryptophan, 3: 1-methyl L-tryptophan, 4: L-tryptophan, 5: Unknown, 6: 5-hydroxy L-tryptophan.

Table 4. Application to Foreign Component Analysis of Nutritious Medicine

\begin{tabular}{lccc}
\hline \hline \multicolumn{1}{c}{ Compounds } & Addition $^{a)} / \mu \mathrm{g}$ & Recovery $^{b)}, \%$ & $\mathrm{RSD}^{c)}, \%$ \\
\hline 5-Methyl L-tryptophan & 0.1 & 95.7 & 2.4 \\
1-Methyl L-tryptophan & 0.1 & 95.3 & 2.7 \\
L-Tryptophan & 10 & 96.0 & 3.4 \\
5-Hydroxy L-tryptophan & 0.1 & 93.0 & 2.5 \\
\hline
\end{tabular}

a) Experimental condition was described in the text. b) Reduced value from extraction percentages. c) Five determinations.

by the proposed method. The electropherogram and results are shown in Fig. 6 and Table 4. Recoveries were calculated based on the percentages of the homogeneous liquid-liquid extraction. The result was satisfactory. The experimental procedure took only $15 \mathrm{~min}$ until sample injection. Compared with other pretreatment methods, the proposed method is simple and convenient.

In conclusion, a two-step separation system was developed combining homogeneous liquid-liquid extraction and a sweeping method. This system is expected to provide not 
only a powerful concentration of extensive analytes but also their selective separation and sensitive determination. Moreover, coupling of an off-line and on-line method gets rid of several kinds of waste in analytical procedure such as sample volume, reagents and time.

\section{References}

1) Sidransky H., Verney E., Cosgrove J. W., Latham P. S., Myeno A. N., Toxicol. Appl. Pharmacol., 126, 108-113 (1994).

2) Molnar-Perl I., Adv. Exp. Med. Biol., 467, 801-816 (1999).

3) Petritis K., Valleix A., Elfakir C., Dreux M., J. Chromatogr. A., 913, $331-340$ (2001).

4) Novatchev N., Holzgrabe U., J. Pharmaceut. Biomed., 26, 779-789 (2001).

5) Chen G., Cheng J., Ye J., Fresenius' J. Anal. Chem., 370, 930-934 (2001).

6) Kaneta T., Shida H., Imasaka T., J. Chromatogra. A, 805, 295-300 (1998).

7) Oshite S., Furukawa M., Igarashi S., Analyst (London), 126, 703-706
(2001).

8) Igarashi S., Yotsuyanagi T., Mikrochim. Acta, 106, 37-44 (1992).

9) Takahashi A., Igarashi S., Sol. Extr. Res. Dev. Jpn., 6, 61—71 (1999).

10) Igarashi S., Takahashi A., Ueki Y., Yamaguchi H., Analyst (London), 125, 797-798 (2000).

11) Quirino J. P., Terabe S., Science, 282, 465-468 (1998).

12) Quirino J. P., Terabe S., J. Chromatogr. A., 856, 465-482 (1999).

13) Quirino J. P., Kim J. B., Terabe S., J. Chromatogr. A, 965, 357-373 (2002).

14) Terabe S., Otsuka K., Ichikawa K., Tsuchiya A., Ando T., Anal. Chem., 56, 111-113 (1984).

15) Terabe S., Trends Anal. Chem., 8, 129-134 (1989).

16) Otsuka K., Quirino J. P., Terabe S., Bunseki Kagaku, 49, 1043-1061 (1999).

17) Otsuka K., Higashimori M., Koike R., Karuhaka K., Okada Y., Terabe S., Electrophoreis, 15, 1280-1283 (1994).

18) Shinoda K., Hato M., Hayashi T., J. Phys. Chem., 76, 909-914 (1972).

19) Takagai Y., Igarashi S., Anal. Bioanal. Chem., 373, 87—92 (2002). 